\title{
BMJ open 'Phaco-ECP': combined endoscopic cyclophotocoagulation and cataract surgery to augment medical control of glaucoma
}

\author{
Dan Lindfield, ${ }^{1}$ Robert W Ritchie, ${ }^{2}$ Michael FP Griffiths ${ }^{1}$
}

To cite: Lindfield D, Ritchie RW, Griffiths MFP, et al. 'Phaco-ECP': combined endoscopic cyclophotocoagulation (ECP) and cataract surgery to augment medical control of glaucoma. BMJ Open 2012;2: e000578. doi:10.1136/ bmjopen-2011-000578

- Prepublication history for this paper is available online. To view these files please visit the journal online (http:// dx.doi.org/10.1136/ bmjopen-2011-000578).

Received 29 December 2011 Accepted 16 April 2012

This final article is available for use under the terms of the Creative Commons Attribution Non-Commercial 2.0 Licence; see http://bmjopen.bmj.com

\footnotetext{
${ }^{1}$ Department of Ophthalmology, Frimley Park Hospital, Camberley, UK ${ }^{2}$ Nuffield Department of Surgical Sciences, John Radcliffe Hospital, Oxford, UK
}

Correspondence to Dr Dan Lindfield; drdanlindfield@gmail.com

\section{ABSTRACT}

Objectives: Does phaco-ECP reduce intraocular pressure? Is phaco-ECP safe?

Design: Retrospective case note review of all patients undergoing phaco-ECP between June 2008 and June 2009. All glaucoma subtypes were included.

Setting: Single District General Hospital Ophthalmology Department within the UK.

Participants: 58 participants case notes reviewed. Mean age 79.0 years $(S D \pm 9.8)$.

Interventions: All patients received combined cataract surgery and endoscopic cyclophotocoagulation.

Outcome measures: Follow-up was 1 day, 1 week, 1 , $3,6,12,18$ and 24 months for intraocular pressure (IOP) measurement. Number of medications, visual acuity and presence of complications were also assessed.

Results: Of the 58 cases performed, 56 case notes $(97 \%)$ were available for analysis. Mean age 79.0 years (SD \pm 9.8$)$. Mean pre-procedural IOP was $21.54 \mathrm{~mm} \mathrm{Hg}(95 \% \mathrm{Cl} 19.86$ to $23.22, \mathrm{n}=56)$. Mean IOP was $14.43 \mathrm{~mm} \mathrm{Hg}(95 \% \mathrm{Cl} 13.65$ to 15.21 , $\mathrm{n}=53)$ at 18 months and $14.44 \mathrm{~mm} \mathrm{Hg}(95 \% \mathrm{Cl} 13.63$ to $15.25, \mathrm{n}=41$ ) at 24 months. The mean drop from baseline to 18 and 24 months was $7.1 \mathrm{~mm} \mathrm{Hg}$. Statistically significant decrease in IOP was demonstrated at all time points $(p<0.001)$. Mean medication usage was 1.97 agents $(95 \% \mathrm{Cl} 1.69$ to 2.25 ) at baseline, 1.96 agents ( $95 \% \mathrm{Cl} 1.70$ to 2.22$)$ at 18 months and 2.07 agents ( $95 \% \mathrm{Cl} 1.76$ to 2.38$)$ at 24 months. No statistically significant change throughout.

Conclusions: This study confirms the safety of phaco-ECP. In this case series, the IOP-lowering effect was significant at all time points; however, the effect of cataract surgery alone was not controlled. A randomised controlled trial is required to draw efficacy conclusions. The authors proposed endoscopic cyclophotocoagulation's main role is to optimise control of low-risk glaucoma of low-risk patients at the time of cataract surgery. However, the authors do not propose that phaco-ECP is a substitute for filtration surgery in high-risk eyes or when low target pressures $(<14 \mathrm{~mm} \mathrm{Hg})$ are indicated.

\section{ARTICLE SUMMARY}

\section{Article focus}

- Is the safety profile of phaco-ECP comparable to other treatment modalities?

- What is the role of the phaco-ECP among other surgical alternatives?

- What effect does ECP in combination with phacoemulsification have on intraocular pressure?

Key messages

- Phaco-ECP is safe.

- ECP combines synergistically with cataract surgery.

- Phaco-ECP reduces intraocular pressure (IOP) between baseline $(21.54 \mathrm{~mm} \mathrm{Hg}, 95 \% \mathrm{Cl} 19.86$ to 23.22 ) and 24 months ( $14.44 \mathrm{~mm} \mathrm{Hg}, 95 \% \mathrm{Cl}$ 13.63 to 15.25$)$ achieving statistical significance at all time points $(p<0.001)$, but the IOP effect achieved by the cataract surgery component of this dual modality treatment is not addressed.

Strengths and limitations of this study

- Data are applicable to typical UK practice and 'early'/'moderate' glaucoma.

- This study suggests that ECP may cause additional IOP lowering than cataract surgery alone, but judgement is reserved until a prospective and controlled study is conducted.

- ECP may have a role outside complex and refractory glaucoma.

\section{INTRODUCTION}

Endoscopic cyclophotocoagulation (ECP) is an emerging tool in the treatment of glaucoma. Endoscopic photoablation of ciliary processes was first described by Shields in $1985 .^{12}$ It was soon apparent that an endoscopic approach resulted in more controllable and focal tissue effects than transscleral 'cyclodiode'. ${ }^{3}$ Diode laser delivery via the transscleral route is inefficient, with only $35 \%$ of laser energy reaching its intended target. The remaining energy causes significant collateral damage to surrounding tissues. ${ }^{4} 5$ 
Presumably due to its similar site of action, ECP has been grouped with transscleral cycloablation and is commonly perceived to inherit the unfavourable side effect profile of its anatomical brethren. The American Academy of Ophthalmology concluded in 2001 that ECP is safer than transscleral therapy ${ }^{6}$ and 10 years later many indications have been suggested for ECP, refractory glaucoma, ${ }^{7} 8$ paediatric glaucoma, ${ }^{9-11}$ aphakic glaucoma ${ }^{9}$ and severe corneal disease. ${ }^{10}{ }^{12}$ All show promise but are only supported by case reports, opinion articles and small case series. However, these subtypes have large variability in disease severity and hence surgical outcomes and account for a small proportion of the glaucoma workload treated by most UK centres.

This paper aims to demonstrate the efficacy and safety combined ECP and cataract surgery (phaco-ECP) to optimise control of 'early' glaucoma for a population representative of the core glaucoma workload in the UK.

\section{MATERIALS AND METHODS}

A retrospective case note audit of all patients undergoing phaco-ECP between June 2008 and June 2009. Patient details were located using theatre records. At time of decision to treat phaco-ECP was offered to optimise intraocular pressure (IOP) control if concurrent 'early' glaucoma (MD $>-6$ decibels, Hodapp Classification) was present at the time of decision to operate for clinically significant cataract. Intraocular pressures of $12 \mathrm{~mm} \mathrm{Hg}$ or higher on medical therapy were considered suitable for ECP with no upper limit. Previous laser trabeculoplasty or glaucoma surgery (trabeculectomy, deep sclerectomy) was not a reason for exclusion. All glaucoma subtypes were included including pseudoexfoliation, pigment dispersion syndrome and narrow angle glaucoma. Ocular hypertension was not considered an indication for ECP.

\section{Follow-up}

Patient were seen following surgical intervention at 1 day, 1 week, 1, 3, 6, 12, 18 and 24 months. Baseline IOP was taken as the pressure measured at decision to treat as this was present for all patients. Mean time between decision to treat and surgery was 7 weeks (range 3-10 weeks). Pressure was measured using a calibrated Goldmann tonometer and continuation of each patient's normal IOP-lowering agents was checked. The presence of complications was assessed at each time point. Observers were not masked to the treatment performed.

\section{Surgical technique}

Cases were performed by three consultant surgeons and two registrars in one moderate-sized unit (district general hospital, 700 beds). All patients received surgery with standardised incisions, viscoelastic and intraocular lens. ECP was performed post-IOL insertion through the main incision and the sideport enlarged to $2.75 \mathrm{~mm}$. The straight endoscopic probe was used (Endo Optiks, Little
Silver, USA) delivering $810 \mathrm{~nm}$ diode laser (Iridex OcuLight SL, Mountain View, California, USA) at $250 \mathrm{~mW}$ and $3500 \mathrm{~ms}$ settings (total 0.875 watts). All processes received a single $3.5 \mathrm{~s}$ exposure. Treatment extent was dependent on access. In all cases, all accessible ciliary processes were photocoagulated hence at least 300 degrees were treated in all cases. Intracameral dexamethasone $0.8 \mathrm{mg} / 0.2 \mathrm{ml}$ (Hospira, Lake Forest, California, USA) was administered to all patients in addition to standard cataract antibiotic prophylaxis (intracameral cefuroxime $1.0 \mathrm{mg} / 0.1 \mathrm{ml}$ ). All patients received standardised postoperative therapy (g. chloramphenicol four times a day for $4 / 52$ and $g$. dexamethasone $0.1 \%$ four times a day for 4 weeks) and instructions to continue their usual preoperative IOP agents.

\section{Statistical analysis}

Data were recorded in an Excel spreadsheet (Microsoft Office 2007; Microsoft Corporation) and then transferred to GraphPad Prism (V.5.0, GraphPad software). The data were analysed for IOP and drop use at each time point. Mean IOP and drop use were calculated together with their 95\% CIs. Multiple comparisons of IOP at all time points were undertaken using repeated measures analysis of variance. Bonferroni's multiple comparison post-test was undertaken to compare pretreatment IOP with IOP at each subsequent time point. The mean differences in IOP with $95 \%$ CIs are given.

Medication use cannot be assumed to follow a Gaussian distribution. Therefore, drop use was analysed using Friedman's test. Using the null hypothesis that no significant difference in drop use exists between time points, Dunn's multiple comparison test was undertaken to compare pre-treatment drop use with drop use at each subsequent time point. p Values $<0.05$ were considered statistically significant throughout.

\section{RESULTS}

Of the 58 cases performed between 1 June 2008 and 1 June 2009, 56 case notes (97\%) were available for analysis. Two records $(3 \%)$ could not be located despite repeated attempts. The mean age of the 22 men (39\%) and 34 women $(61 \%)$ was 79.0 years (SD \pm 9.8 years). Mean follow-up was 22 months ( \pm 3.2 months) (range 12-24 months). Three patients $(5 \%)$ had pseudoexfoliation, one patient (2\%) had pigment dispersion syndrome and four patients $(7 \%)$ were narrow-angle subtype. The remaining 48 patients $(86 \%)$ were primary open-angle glaucoma. No normal tension patients were in the cohort. Previous glaucoma intervention included two patients (4\%) whom had previous argon laser trabeculoplasty. None had previous filtration surgery.

\section{IOP outcomes}

Table 1 documents IOP outcomes over the study time points and is demonstrated graphically in figure 1. Mean pre-procedural IOP was $21.54 \mathrm{~mm} \mathrm{Hg}(95 \%$ CI 1.68 , $\mathrm{n}=56$ ). A statistically significant decrease in IOP 


\begin{tabular}{|c|c|c|c|c|c|c|c|c|c|}
\hline IOP & Pre & 1 Day & 1 Week & 1 Month & 3 Months & 6 Months & 12 Months & 18 Months & 24 Months \\
\hline Mean (mm Hg) & 21.54 & 16.44 & 14.61 & 14.91 & 14.12 & 13.98 & 13.87 & 14.43 & 14.44 \\
\hline $95 \% \mathrm{Cl}$ & 1.68 & 1.33 & 1.04 & 1.25 & 0.83 & 0.73 & 0.81 & 0.78 & 0.81 \\
\hline \multicolumn{10}{|c|}{ Analysis of variance } \\
\hline $\begin{array}{l}p \text { Value versus } \\
\text { pre-treatment }\end{array}$ & & $<0.0001$ & $<0.0001$ & $<0.0001$ & $<0.0001$ & $<0.0001$ & $<0.0001$ & $<0.0001$ & $<0.0001$ \\
\hline$\underline{n}$ & 56 & 54 & 54 & 44 & 49 & 55 & 55 & 53 & 41 \\
\hline
\end{tabular}

compared with pre-procedural IOP was demonstrated at all time points, beginning at 1 day following treatment. The mean IOP at 18 and 24 months was $14.43 \mathrm{~mm} \mathrm{Hg}$ $(95 \%$ CI $0.78, \mathrm{n}=53)$ and $14.44(95 \%$ CI $0.81, \mathrm{n}=41)$, respectively. The mean difference in IOP from baseline to 18 and 24 months was $7.1 \mathrm{~mm} \mathrm{Hg}(\mathrm{p}<0.001)$.

Surgical failure was defined as IOP $>21 \mathrm{~mm} \mathrm{Hg}$ or not reduced by at least $20 \%$ for two consecutive visits from 3 months onwards. A Kaplan-Meier survival curve (figure 2) plots survival over time. At 24 months, survival was $76 \%$.

\section{Medication outcomes}

The analysis of intraocular pressure-lowering medications at each time point is shown in table 2 and is demonstrated graphically in figure 3 . The number shown represents the number of topical IOP-lowering agents not the number of bottles used. For example, fixed prostaglandin and $\beta$ blocker combinations were recorded as two medications. Preoperative mean medication usage was 1.97 agents (95\% CI 0.28). At 18 and 24 months, respectively, the mean number of topical agents was 1.96 (95\% CI 0.26) and 2.07 (95\% CI 0.31), with no statistically significant change in agent use compared with pre-treatment. No patients $(0 \%)$ received oral or intravenous acetazolamide at any point.

\section{Visual acuity outcomes}

No patients $(0 \%)$ lost best corrected visual acuity at 3, 6 , 12, 18 or 24 months compared with baseline.

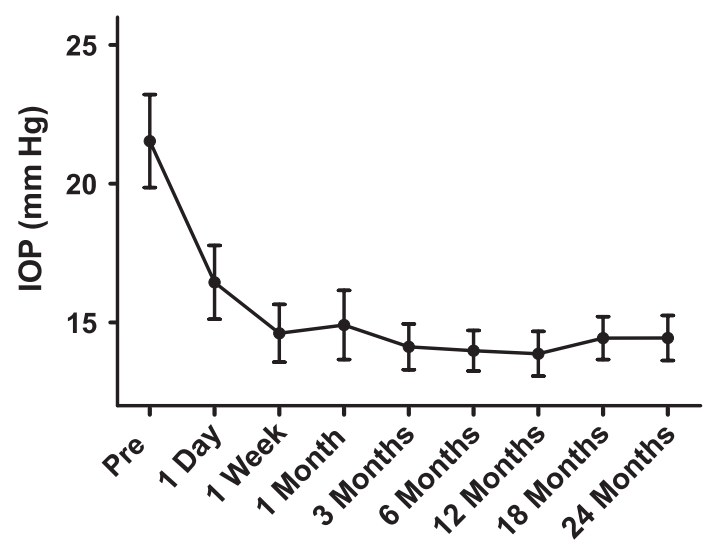

Figure 1 Mean intraocular pressure (IOP) over time with $95 \%$ Cls.

\section{Complications}

Fifty-three cases $(95 \%)$ had no complication. There was one case $(2 \%)$ of steroid-related raised IOP at 1 month (19 $\mathrm{mm} \mathrm{Hg}$ at week 1 to $30 \mathrm{~mm} \mathrm{Hg}$ at month 1 ), which returned to baseline upon cessation of steroid. One case (2\%) had vitreous in the anterior chamber noted on the first postoperative day with a peaked pupil. The postulated mechanism was a small area of zonular dehiscence not noted during surgery. There was no intraocular lens tilt, IOL decentration or IOP rise. Nd:YAG was applied to the strand and vision remained at 6/7.5. There were three cases $(5 \%)$ of anterior uveitis postoperatively, defined as anterior chamber cells of two-plus or greater but no cases of fibrinous uveitis. Visually acuity in these cases was 6/6 (one case) and 6/9 (two cases). There were no cases of hyphaema, hypotony, lens instability or dislocation, cystoid macular oedema, retinal tears or endophthalmitis.

\section{DISCUSSION}

\section{Phaco-ECP safety}

The safety profile of ECP in the existing literature is varied and related to the disease subtype it is employed to treat. It is not relevant to include safety data from small case series involving complex or refractory patients. These procedures are certainly subject to a less favourable side effect profile than ECP when combined with phaco. ${ }^{13}$

The literature assessing the safety of only ECP in combination with phaco is scarce. One small study

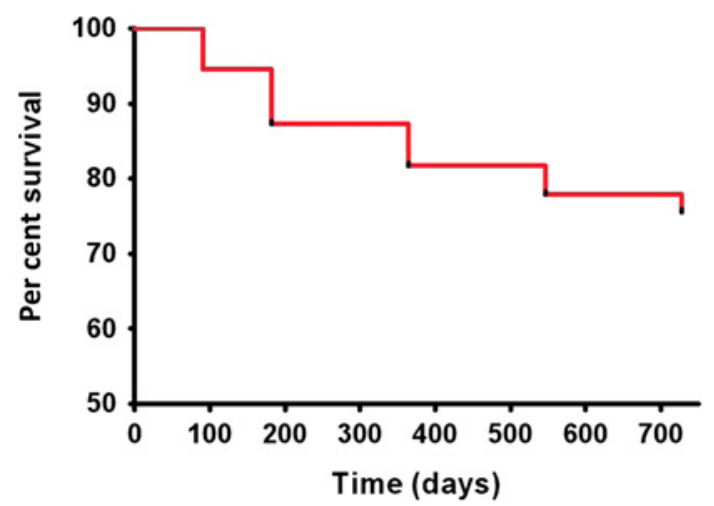

Figure 2 Kaplan-Meier survival curve. Failure = "intraocular pressure 21 or $<20 \%$ drop from baseline." 

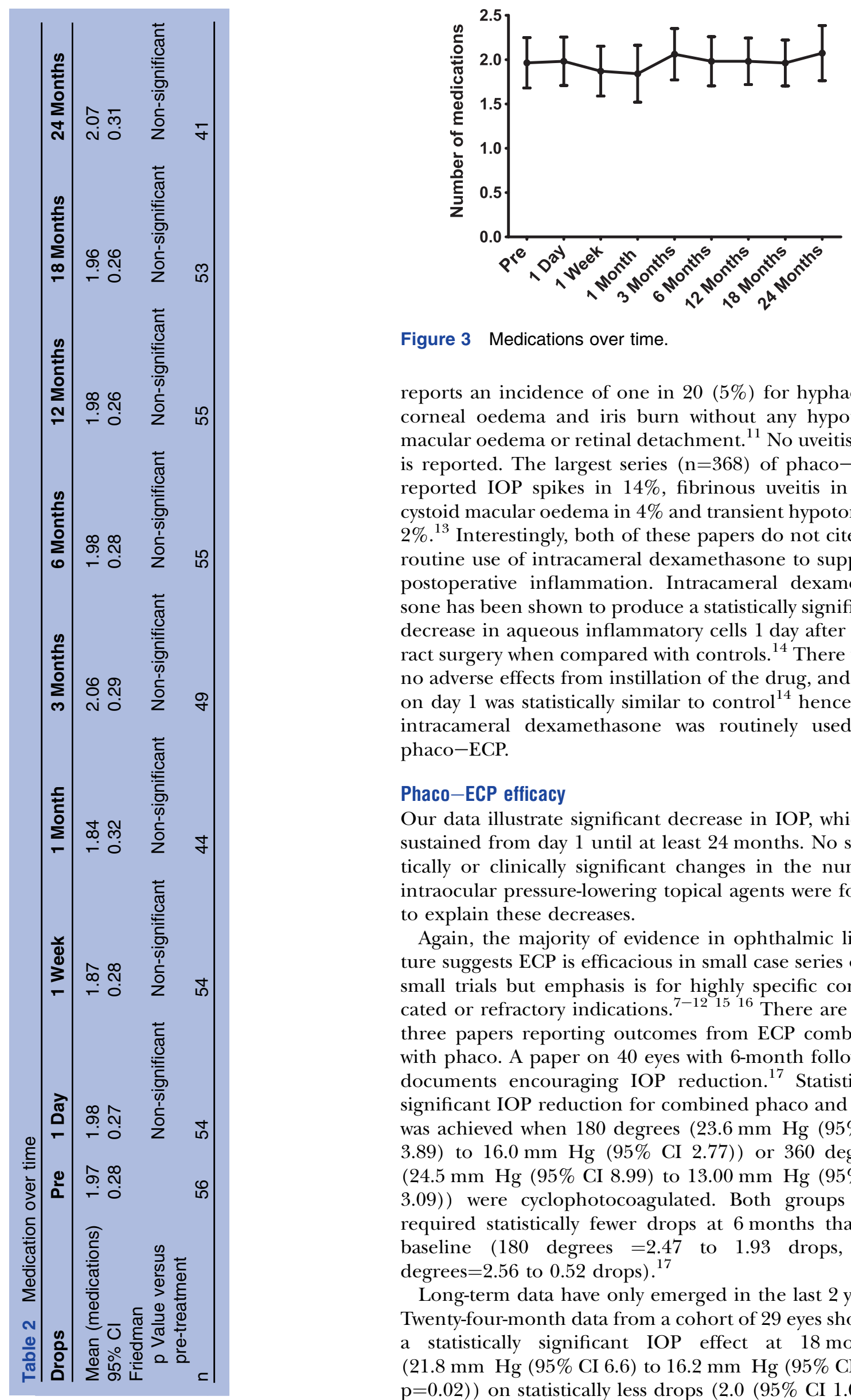

Figure 3 Medications over time.

reports an incidence of one in $20(5 \%)$ for hyphaema, corneal oedema and iris burn without any hypotony, macular oedema or retinal detachment. ${ }^{11}$ No uveitis rate is reported. The largest series $(n=368)$ of phaco-ECP reported IOP spikes in 14\%, fibrinous uveitis in $7 \%$, cystoid macular oedema in $4 \%$ and transient hypotony in $2 \%{ }^{13}$ Interestingly, both of these papers do not cite the routine use of intracameral dexamethasone to suppress postoperative inflammation. Intracameral dexamethasone has been shown to produce a statistically significant decrease in aqueous inflammatory cells 1 day after cataract surgery when compared with controls. ${ }^{14}$ There were no adverse effects from instillation of the drug, and IOP on day 1 was statistically similar to control ${ }^{14}$ hence why intracameral dexamethasone was routinely used for phaco-ECP.

\section{Phaco-ECP efficacy}

Our data illustrate significant decrease in IOP, which is sustained from day 1 until at least 24 months. No statistically or clinically significant changes in the number intraocular pressure-lowering topical agents were found to explain these decreases.

Again, the majority of evidence in ophthalmic literature suggests ECP is efficacious in small case series or in small trials but emphasis is for highly specific complicated or refractory indications. ${ }^{7-12} 1516$ There are only three papers reporting outcomes from ECP combined with phaco. A paper on 40 eyes with 6-month follow-up documents encouraging IOP reduction. ${ }^{17}$ Statistically significant IOP reduction for combined phaco and ECP was achieved when 180 degrees $(23.6 \mathrm{~mm} \mathrm{Hg}(95 \%$ CI 3.89 ) to $16.0 \mathrm{~mm} \mathrm{Hg}$ (95\% CI 2.77)) or 360 degrees (24.5 mm Hg (95\% CI 8.99) to $13.00 \mathrm{~mm} \mathrm{Hg} \mathrm{(95 \%} \mathrm{CI}$ 3.09)) were cyclophotocoagulated. Both groups also required statistically fewer drops at 6 months than at baseline $(180$ degrees $=2.47$ to 1.93 drops, 360 degrees $=2.56$ to 0.52 drops $).{ }^{17}$

Long-term data have only emerged in the last 2 years. Twenty-four-month data from a cohort of 29 eyes showed a statistically significant IOP effect at 18 months (21.8 $\mathrm{mm} \mathrm{Hg}(95 \%$ CI 6.6) to $16.2 \mathrm{~mm} \mathrm{Hg}(95 \%$ CI 4.1, $\mathrm{p}=0.02))$ on statistically less drops $(2.0(95 \%$ CI 1.0$)$ to 
$0.9(95 \%$ CI $0.9, \mathrm{p}=0.04))$. However, statistical significance was not reached at 24 months $(17.9 \mathrm{~mm} \mathrm{Hg}(95 \%$ CI 4.9) on 1.2 (95\% CI 0.8) drops, $\mathrm{p}=0.18)$. Notably, this cohort contained a mixed bag of subtypes including PXF, normal tension and narrow angle glaucoma. ${ }^{11}$

Most recently, a Brazilian paper documents results from 368 eyes with follow-up exceeding 2 years. ${ }^{13}$ Statistical significance $(p \leq 0.001)$ was reported at all time points comparing IOP at baseline $(23.1 \mathrm{~mm} \mathrm{Hg}, 95 \%$ CI $5.6)$ to 12 months $(12.2 \mathrm{~mm} \mathrm{Hg}, 95 \%$ CI 2.19), 24 months (12.14 mm Hg, 95\% CI 2.9) and last appointment $(12.3 \mathrm{~mm} \mathrm{Hg}$, 95\% CI 2.44, mean followup 35.2 months SD 8.1). ${ }^{13}$ Furthermore, there was a statistically significant reduction in medication from 1.44 (95\% CI 0.97$)$ drops preoperatively to 0.37 (95\% CI $0.68)$ at 2 years $(\mathrm{p} \leq 0.001$ for all time points). IOP reductions of the magnitude seen from this report ${ }^{13}$ were not reproduced in our series presumably due to the differences in pigmentation-Brazilian eyes are more pigmented, absorb more laser energy and hence should illustrate a greater IOP drop side effects in our series were less common, which may again be explained by differences in laser energy uptake.

The IOP effect of cataract surgery alone? A difficult question It is well documented that cataract surgery confers a pressure reduction in eyes with or without glaucoma. ${ }^{15} 1618$ However, this benefit is variable and dependent on starting IOP and type of glaucoma. For example, in narrow angle glaucoma, cataract extraction alone has been shown to normalise $\mathrm{IOP}^{19}$ and this effect can be attributed anatomically to the removal of a large and anteriorly positioned lens and therefore removal of outflow resistance. These eyes also have higher starting pressures so some of the perceived effect could be due to regression towards the mean. ${ }^{18}$ The effect of lens removal in open-angle glaucoma is less clear and the exact mechanism is yet to be elucidated. ${ }^{16}$ By definition, in open-angle glaucoma, the trabecular meshwork is unobstructed and most studies suggest a drop in IOP after cataract surgery is only transient. Reported IOP changes ranges from a reduction of $8.5 \mathrm{~mm} \mathrm{Hg}$ in eyes with a high starting pressure $(24-29 \mathrm{~mm} \mathrm{Hg})$ to a $1.7 \mathrm{~mm} \mathrm{Hg}$ rise in IOP in low-pressure eyes $\left(14 \mathrm{~mm} \mathrm{Hg}\right.$ or less). ${ }^{20}$ Duration of effect is also contentious. Effects lasting 12, 18 or 24 months are commonly reported,${ }^{16}{ }^{18}$ but continued response is rare. Meta-analysis in 2002 revealed a $2-4 \mathrm{~mm} \mathrm{Hg}$ pressure drop from cataract surgery with effect diminishing after $18-24$ months, ${ }^{21}$ but more recent papers with longer follow-up illustrated a drop in IOP of approximately $3 \mathrm{~mm} \mathrm{Hg}$ with $75 \%-85 \%$ of patients maintaining this IOP reduction at 5 years. ${ }^{20}{ }^{22-25}$ Many criticisms are levelled at the attribution of cause and effect within these papers. ${ }^{18}$ A 2011 review of the current evidence concludes: "the mild and variable nature of any IOP decrease from lens extraction suggests a marginal benefit only in milder forms of POAG. There is currently no evidence of any quality to suggest that lens extraction routinely represents a clinically useful treatment for primary open angle glaucoma."16

The symbiotic combination of cataract surgery and ECP

ECP combines almost symbiotically with cataract surgery. Glaucoma like cataract is predominantly a disease of the older population and is often concurrent. Early ECP necessitated a pars plana approach with its associated and additional posterior pole risks ${ }^{1}$ but can now be performed through standard phaco incisions. Anatomical access to the ciliary processes is improved by lens removal, and the instrumentation and viscoelastics required are inherent to both procedures. The procedure adds $5 \mathrm{~min}$ to the on-table time. One of the much stated risks of ECP over transscleral or trabecular laser therapy - the need to enter the eye and thus impose possible infectious complications-is also a moot point with combined surgery.

\section{Limitations of the study}

This study represents a retrospective analysis of intraocular pressure, complication and medication outcomes. The data are therefore less robust than prospective collection, but common limitations for retrospective data of bias and confounding were minimised as all patients receiving surgery within the 12-month sampling period were included and only two of 58 case notes were not available. Visual field analysis was also not included. Any change in field parameters is inextricably linked with lens-related factors in addition to any possible glaucomatous change and very difficult to separate.

The pressure-lowering effect of the ECP component of phaco-ECP is difficult to isolate. Cataract surgery alone lowers IOP but the degree of response is multifactorial (angle depth, starting IOP) and duration rarely persists beyond 12-24 months. A prospective trial comparing phaco-ECP with phaco-only controls is required to truly answer the question. The IOP effect of phaco-ECP in this study is greater than most published studies assessing phaco alone, but direct comparison is neither wise nor possible.

\section{CONCLUSIONS}

The combination of ECP with phacoemulsification shows promise when applied a wide patient population of mixed-subtype early glaucoma. We, however, do not propose that phaco-ECP is a substitute for filtration surgery in high-risk eyes, rapidly progressive patients or when a low target pressure $(<14 \mathrm{~mm} \mathrm{Hg})$ is indicated. The results of this study show transposable and safe results from ECP that can be applied to current UK glaucoma practice but do not entangle the pressurelowering effect of cataract surgery alone. Until a randomised controlled trial compares phaco alone and phaco-ECP, its role outside complex refractory glaucoma is anecdotal but shows promise.

Contributors DL conceived, designed and acquired the data, drafted the article and approved the final manuscript. RWR analysed and interpreted the data, 
revised the manuscript and approved the final version. MFPG was involved in the conception of the article, revising the manuscript and its final approval.

Competing interests None.

Ethics approval Retrospective audit of current standard practice.

Provenance and peer review Not commissioned; externally peer reviewed.

Data sharing statement There are no additional data available.

\section{REFERENCES}

1. Shields MB. Intraocular cyclophotocoagulation. Trans Ophthalmol Soc U K 1986;105:237-41.

2. Shields MB. Cyclophotocoagulation surgery for glaucoma: past, present, and future. Trans Am Ophthalmol Soc 1985;83:285-303.

3. Shields MB, Chandler DB, Hickingbotham D, et al. Intraocular cyclophotocoagulation. Histopathic evaluation in primates. Arch Ophthalmol 1985;103:1731-5.

4. Bloom PA, Dharmaraj S. Endoscopic and transscleral cyclophotocoagulation. Br J Ophthalmol 2006;90:666-8.

5. McKelive PA, Walland MJ. Pathology of cyclodiode laser: a series of nine enucleated eyes. Br J Ophthalmol 2002;86:381-6.

6. Pastor SA, Singh K, Lee DA, et al. Cyclophotocoagulation: a report by the American Academy of Ophthalmology. Ophthalmology 2001:108:2130-8.

7. Lima FE, Magacho L, Carvalho DM, et al. A prospective, comparative study between endoscopic cyclophotocoagulation and the Ahmed drainage implant in refractory glaucoma. J Glaucoma 2004;13:233-7.

8. Yu MB, Huang SS, Ge J, et al. The clinical study of endoscopic cyclophotocoagulation on the management of refractory glaucoma (In Chinese). Zhonghua Yan Ke Za Zhi 2006;42:27-31.

9. Carter BC, Plager DA, Neely DE, et al. Endoscopic diode laser cyclophotocoagulation in the management of aphakic and pseudophakic glaucoma in children. $J$ AAPOS 2007;11:34-40.

10. Al-Haddad CE, Freedman SF. Endoscopic laser cyclophotocoagulation in pediatric glaucoma with corneal opacities. J AAPOS 2007;11:23-8.

11. Yip LW, Yong SO, Earnest A, et al. Endoscopic cyclophotocoagulation for the treatment of glaucoma: an Asian experience. Clin Experiment Ophthalmol 2009;37:692-7.

12. Lee RM, Al Raqqad N, Gomaa A, et al. Endoscopic cyclophotocoagulation in osteo-odonto-keratoprosthesis (OOKP) eyes. J Glaucoma 2011;20:68-9.

13. Lima FE, Carvalho DM, Avila MP. Phacoemulsification and endoscopic cyclophotocoagulation as primary surgical procedure in coexisting cataract and glaucoma (In Portuguese). Arq Bras Oftalmo 2010;73:419-22.

14. Chang DT, Herceg MC, Bilonick RA, et al. Intracameral dexamethasone reduces inflammation on the first postoperative day after cataract surgery in eyes with and without glaucoma. Clin Ophthalmol 2009;3:345-55.

15. Jamil AZ, Iqbal K, Ur Rahman F, et al. Effect of phacoemulsification on intraocular pressure. J Coll Physicians Surg Pak 2011;21:347-50.

16. Walland MJ, Parikh RS, Thomas R. There is insufficient evidence to recommend lens extraction as a treatment for primary open angle glaucoma: an evidence based perspective. Clin Experiment Ophthalmol. Published Online First: 13 January 2011. doi:10.1111/ j.1442-9071.2011.02617.x

17. Kahook MY, Lathrop KL, Noeckler RJ. One-site versus two-site endoscopic cyclophotocoagulation. J Glaucoma 2007;16:527-30.

18. Shrivastava A, Singh K. The effect of cataract extraction on intraocular pressure. Curr Opin Ophthalmol 2010;21:118-22.

19. Wishart PK, Atkinson PL. Extracapsular cataract extraction and posterior chamber lens implantation in patients with primary chronic angle-closure glaucoma: effect on intraocular pressure control. Eye (Lond) 1989;3:706-12.

20. Poley BJ, Lindstrom RL, Samuelson TW, et al. Intraocular pressure reduction after phacoemulsification with intraocular lens implantation in glaucomatous and nonglaucomatous eyes: evaluation of a causa relationship between the natural lens and open-angle glaucoma. J Cataract Refract Surg 2009;35:1946-55.

21. Liu DT, Lee VY, Chiu TY, et al. Long-term intraocular pressure control after clear corneal phacoemulsification in glaucoma patients. J Cataract Refract Surg 2006;32:183.

22. Friedman DS, Jampel HD, Lubomski LH, et al. Surgical strategies for coexisting glaucoma and cataract: an evidence-based update. Ophthalmology 2002;109:1902-13.

23. Kim DD, Doyle JW, Smith MF. Intraocular pressure reduction following phacoemulsification cataract extraction with posterior chamber lens implantation in glaucoma patients. Ophthalmic Surg Lasers 1999;30:37-40.

24. Shingleton BJ, Gamell LS, O'Donoghue MW, et al. Long-term changes in intraocular pressure after clear corneal phacoemulsification: normal patients versus glaucoma suspect and glaucoma patients. J Cataract Refract Surg 1999; 25:885-90.

25. Shingleton BJ, Pasternack JJ, Hung JW, et al. Three and five year changes in intraocular pressures after clear corneal phacoemulsification in open angle glaucoma patients, glaucoma suspects, and normal patients. J Glaucoma 2006;15:494-8. 